\title{
Design on Interface Circuit of Simulation Training Evaluation subsystem for an Antiaircraft Missile hardware-in-loop simulator
}

\author{
LI Ke LI Yan Jiang Huixia HE Xin \\ 4th department, Shijiazhuang Mechanical Engineering College, Shijiazhuang, HEBEl, China, \\ 050003 \\ Ken_like@126.com
}

Keywords: antiaircraft missile; interface circuit of simulation training evaluation subsystem; interface circuit

\begin{abstract}
A simulation training evaluation subsystem can't be ignored when an antiaircraft missile simulator is developed whatever type it is. Otherwise, the result of simulation training couldn't be verified. So one of key difficulties is how to design and realize its Interface Circuit when an antiaircraft missile hardware-in-loop simulator is implemented. Interface circuit of simulation training evaluation subsystem provides quantitative data for the evaluating subsystem. By means of quantitative data sampled, the evaluating system could record and analyze whether operations is right or not. The interface circuit, whose kernel component is I/O card PCL-722, is designed combined with exploitation of one antiaircraft missile hardware-in-loop simulator. Experiments show that the design of the mentioned interface circuit is feasible and effective.
\end{abstract}

\section{Introduction}

To meet the demand of antiaircraft missile troops daily combat operation training and evaluation, technical training etc. Let weapon system users form their combat effectiveness as soon as possible, the paper takes full account of air-defense force basic and practical training demand, for example, their objective conditions, convenience and other factors. With computer simulation, computer network technology, an antiaircraft missile hardware-in-loop simulator is developed. A simulation training evaluation subsystem can't be ignored when an antiaircraft missile simulator is developed whatever type it is. Otherwise, the result of simulation training couldn't be verified. So one of key difficulties is how to design and realize its Interface Circuit when an Antiaircraft Missile hardware-in-loop simulator is implemented.

2 An antiaircraft Missile hardware-in-loop simulator hardware ${ }^{[1,2]}$

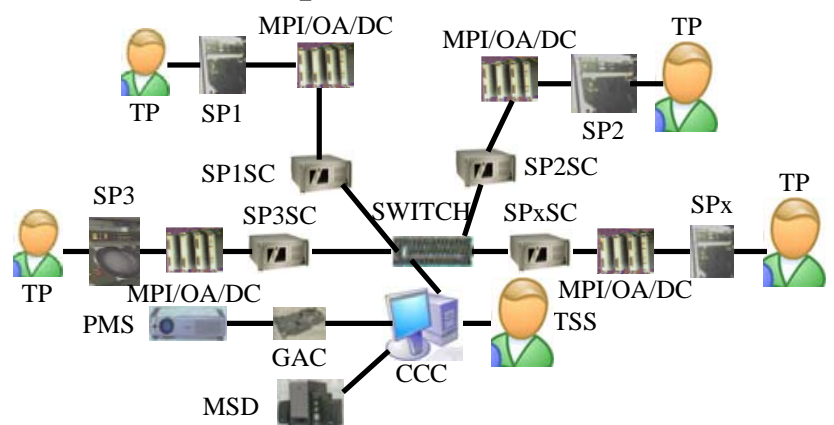

CCC: Central control computer PMS: Projection machine and screen SP: simulating panel MSD: Multimedia sound devices GAC: Graphic accelerative card TP: Trained person MPI/OA/DC: Multipath I/O and A/D card TSS: Training and simulation supervisor SP1, 2, 3, x SC: SP1, 2, 3, x sample computer

Fig.1 the hardware topological structure of an antiaircraft missile hardware-in-loop simulator 
According to the specific environment and conditions which are confronted by an antiaircraft Missile hardware-in-loop simulator, its hardware architecture is designed as a star architecture of LAN, that fully embodies modular, universal, standardized, open and other idea. So the antiaircraft Missile hardware-in-loop simulator is easy to develop and maintain. Conventional PC is selected according to the demand of each simulation entity and for cutting cost and convenience. These PCs connect with and control other hardware resources of one simulation entity of the simulator; they are connected and realized communication over the network. The topology of the antiaircraft missile hardware-in-loop simulator is shown as Figure 1.

\section{Evaluation Idea and Methods ${ }^{[3,4]}$}

Combination of qualitative and quantitative evaluation. To be able to assess the trainees Comprehensive subjective and objective aspects of training results, the simulator adopts an evaluation idea, which is also combination of qualitative and quantitative assessment. The evaluation index system established includes not only quantitative factors, for example, operational process, time and etc., which could be sampled and analyzed, but also including the qualitative factors, for example, trainees' morale, psychology and etc. which couldn't be sampled by machine now and could just be evaluated by training and simulation supervisor.

Integrated usage of the Delphi method, AHP method, expert scoring method and fuzzy comprehensive evaluation method as a concrete application method. Delphi method is used to determine the choice of the index system of evaluation indicators; The AHP method is used to determine the weights of the index system of evaluation indicators; Expert scoring method is used to evaluate training personnel subjective mental quality by training and simulation supervisor; Finally the fuzzy comprehensive evaluation is used to get a final assessment goal of one training effect.

\section{Requirements Analysis ${ }^{[3,4]}$}

The operational flow of a tactical antiaircraft missile hardware-in-loop simulator applied to training should be as same as that of the real equipment simulated. General operational flow of a tactical antiaircraft missile is as follow, manipulators make decision by synthesizing director's command and information from instruments and image, and they manipulate various pilot levers or buttons of panels. Different information is processed by diversified data handling systems; different relevant information is displayed on display systems or instruments. Through analyzed, all of manipulating actions could be divided into two categories:

Discrete action. It is indicated to action by buttons and switches of panels, for example, pressing launch button, screwing antenna switch etc. The possible wrong manipulations are perversion and repeat. Under condition of equipment hardware fault-free, if only a key (or button) has moved, it is considered to have one operational conduct and to result in condition change (perhaps one button from "opening” to "shutting” or one key from "rotating” to "braking”).

Analogical action. It is indicated to action by various pilot levers, and including a few operations of some buttons and knobs, for example, pressing one key 5 seconds until one indicator light blanking. The possible mistaken operations are wrong operational results, perversion and repeat. For instance, one key was pressed but pressing time isn't in accordance with time required or one key was rotated but the parameter wasn't the index required. Though this kind of manipulations has been done, condition change might not happen.

\section{Interface Circuit of Simulation Training Evaluation Subsystem}

By means of previous analysis, the logical structure of the interface circuit of simulation training evaluation subsystem is shown as Figure 2. The principle of the interface circuit of simulation training evaluation subsystem is as follow: all of operational signals, which are created by trainee's 
manipulating various operational buttons of one simulating panel including switches and other components, are transferred to multiple I/O cards to form binary sampled words having a defined bits. A sample computer scans interface between $\mathrm{I} / \mathrm{O}$ card and it to get output at regular intervals, then the sampled words acquired by a SPxSC could be transferred into central control computer through switch, and then central control computer stores the sampled words into sampled data database. By comparing change of each bit of the sampled words on different sampling time and the flow of the sampled words with standardized operational flow of the equipment, trainee's operational quantitative data could be recorded and assessed, and his simulation training effect could be assessed too.

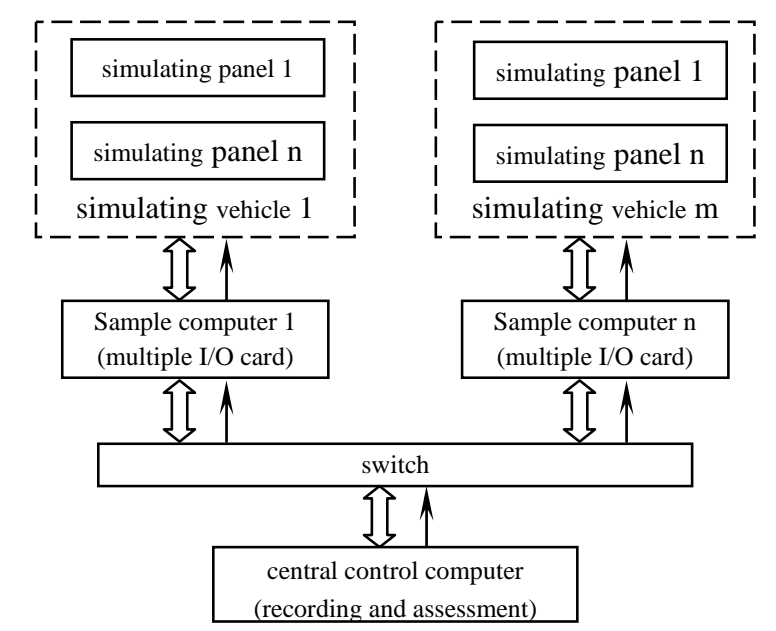

Fig.2 the interface circuit of simulation training evaluation subsystem

\section{Complement of interface circuit with PCL722 card}

The paper selects PCL722 card manufactured by EVOC Company. It is a 144-channel parallel digital I/O card. The card offers two operating modes, 144 and 96 . The switch of two modes can be switched by setting a jumper situation on the card. According to the mounting panel switch class statistics, the 144 mode is selected.

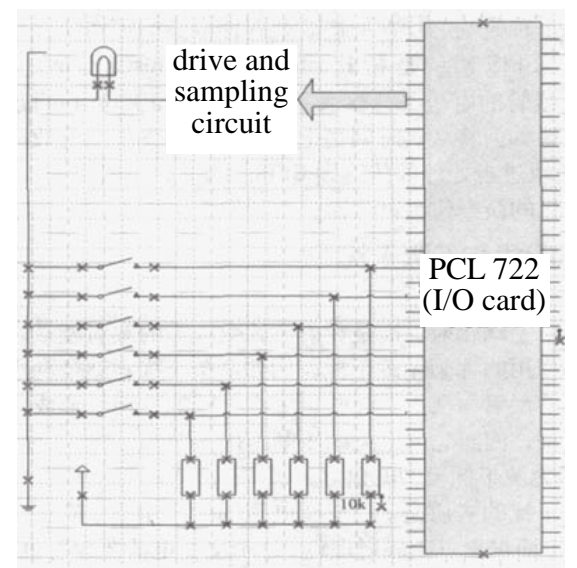

Fig.3 schematic diagram of interface circuit based on PCL722 card

As figure 3, the digital I/O card PCL722 samples situations of each switch by sampling circuit with the form of high or low logical voltage(for example TTL), that is called its I function. So the situations of each switch could be monitored in time. In general, the situations of all switches are in the "off" state and the sampled point voltages are high. The bits of one sampled word inputted into sample computer through PCL722 are logical "1." If a switch is closed, the voltage of the relevant sampled point is from high to low, and then the sampling circuit receives a low logical voltage, one sampled word inputted into sample computer through PCL722 is changed too as one of bits of it is 
changed from 1 to 0 . In this way, the bits change of one binary sampled word inputted into central control computer could be reflected the situation of one switch on one simulating panel, and the central control computer gets information for evaluation.

\section{Selection on network transfer protocol ${ }^{[5]}$}

There are two main protocols of network in the transfer layer: connect-oriented protocol and connectionless protocol. Connect-oriented protocol is called TCP, connectionless protocol is called UDP. TCP provides reliable errorless data transmission between two computers; it will establish a virtual connection between the source computer and the destination computer when TCP is applied. Once the connection is established, data between two computers could be transferred by two-way stream of bytes. UDP provides a connectionless data transfer service, which is an unreliable but most efforts for data transfer. It does not guarantee the transmission of data packets, and the order of received data packets can't be restored erroneously, and data packet loss can't be found too, amendment could be done only by retransmission. According to demand of the antiaircraft missile hardware-in-loop simulator, TCP is selected as data transfer protocol and communication between central control computer and control computers is realized through the console Winsock of VB. It could be simple to implement TCP function call network services with the console Winsock of VB.

\section{Conclusions}

The interface circuit is a bridge between training and assessment of the antiaircraft missile hardware-in-loop simulator. Whether it is good or not to design the interface circuit could have direct impact on simulation effect of the simulator. Experiments show that the interface circuit with PCL722 card introduced in the paper is feasible and effective.

\section{References:}

[1] Zhang Wang Wei, Meng Chen study the overall structure of large-scale air missile simulation training system [J] Ordnance Industry Automation, 2008,3 (7): 4421.

[2] Li Ke, Wei Baohua, Zheng Silong,etc. Design on Half Hardware-in-loop simulation of Training and Simulation System of One antiaircraft Missile [J]. Computer Simulation, 2008,25.

Han Hongyan, Cuishao Hui, Li Ke. Battle SAM Evaluation of HILS training [J].

Computer Applications, 2008, (S).

[3] Han Hongyan, Cuishao Hui, Li Ke. Battle SAM Evaluation of HILS training [J]. Application Reserch of Computer , 2008, (25).

[4] Han Hongyan. A certain type of ground simulation training in assessment technology research and application [D]. Ordnance Engineering College master's degree thesis, 2008.

[5] HU Xiaoqiang, DAI Hang. COMPUTER NETWORK [M], BEI UNIVERSITY OF POSTS AND TELECOMMUNICATIONS PRESS, 2005.9 